and the environment than appeared to be the case with the antiscorbutic vitamin. In the second lecture of the first publication I stated :-

"For we know something of the part played by accessory food factors in such deficiency diseases as beri-beri and scurvy, and we know something of the part played by these substances in growth, but in the case of rickets we are apparently up against a combination of both a deficiency disease and growth, rickets, in fact, being a disease accompanying growth."

Again, in the same lecture I stated:-

"Since, further, the dietetic problem is one of balance, foodstuffs which contain no anti-rachitic factor cannot be considered as neutral, but as positively rickets-producing, for the more of them that is eaten the greater is the necessity for foods containing the factor. It is probable that bread is the worst offender."

Even the effect of exercise and its relation to the dietetic theory of rickets I mentioned in the first publication and discussed at greater length in the second publication, ${ }^{2}$ and the views on this point that $I$ advanced then are substantially the same as I hold to-day. How near all these ideas approximate to my later views can be seen in my last and more complete publication on the subject of rickets-viz., Special Report Series, No. 61, Medical Research Council.

Research has only touched the edge of the subject, and the amazing amount of work on rickets now being started in America and elsewhere may soon necessitate modification and extension of my published views as to the process of events occurring in this disease. It is possible that the deductions made from my experimental work on rickets may prove absurd, but $\bar{I}$ am not convinced that this has yet happened, nor have I seen any reason to abandon my earlier published views. I am, Sir, yours faithfully,

EDWARD MELLANBY.

The University, Sheffield, March 28th, 1922.

\section{PROSTATECTOMY AT ADVANCED AGE.}

To the Editor of THE LANCET.

SIR,-With reference to Mr. J. B. Macalpine's article in your last issue on successful prostatectomy at 90 years, in which he states that he has not been able to find in the literature a record of a prostatectomy at so advanced an age, I would like to give a brief record of a patient operated on by myself. He was an old hunting man well advanced in his ninety-first year, and his vigorous constitution was illustrated by the fact that he was accustomed to sing "John Peel " in a lusty voice every morning in his bath. For some years he had been troubled with irritability of the bladder and dribbling. One day he was seized with acute retention of urine. A catheter was passed and he stated that he had never been so insulted in his life and refused to have such a thing done again. He said that he had heard an operation could be performed for this condition and told his medical adviser to send for a surgeon at once. I was called in and performed a prostatectomy in two stages. He healed up satisfactorily in five weeks from the first operation without any complications. Thereafter he could pass his water with perfect ease and control, and was able to return to his hunting. He died of influenzal pneumonia some two years after his operation. Some men at the age of 90 are more vigorous and better fitted to stand an operation than many men of 75 .

I am, Sir, yours faithfully,

Harley-st., W., March 31st, 1922. FRANK KIDD, M.Ch.

Ta the Editor of NHE LANCET.

SIR,-OOn Feb. 27th, 1920, I performed the operation of prostatectomy on a man aged 89 years and 2 months, in the Petersfield Cottage Hospital. It could not be considered a favourable case. The patient had been catheterised for some days and was sent into hospital because an instrument could no longer be passed though several forms were tried. On admission the bladder was found distended, nearly reaching the umbilicus, and relief was urgent. The patient was anxious for anything to be done to relieve his pain and left himself in our hands. The first thought, owing to the patient's great age and feeble condition, was suprapubic drainage. Mr. R. G. Cross administered the anæesthetic, and the patient took this so well that it seemed a pity not to do a total enucleation when we had the bladder open. Subsequent events justified the decision, for he made an uneventful recovery and is now, more than two years after the operation, in good health, free from urinary trouble and able to get about.

$$
\text { I am, Sir, yours faithfully, }
$$

Petersfield, Hants, April 4th, 1922.

$$
\text { W. P. PANCKRIDGE. }
$$

\section{B-VITAMIN AND PNEUMOCOCCAL INFECTION.}

\section{To the Editor of THE LANCET.}

SIR,-Though the organismal theory of beri-beri is now of historical interest only, there is still no satisfactory explanation of the frequency with which bacteria attack the tissues of animals living on a diet deficient in B-vitamin. In an attempt to determine the exact nature of the rupture in the defence mechanism resulting from a deficiency of vitamin $B$, experiments were carried out on pigeons with the pneumococcus. As is well known, the pigeon is practically immune to the action of the pneumococcus even when it is introduced intraperitoneally in relatively enormous doses. In normal pigeons the pneumococci have completely disappeared from the peritoneal cavity in from three to eight hours after the injection. The cause of this destruction of pneumococci has not been satisfactorily explained, though there is some evidence to show that the pneumococcus is rapidly killed at 107-108 ${ }^{\circ} \mathrm{F}$., temperatures which, of course, are normal for the healthy pigeon. Strouse ${ }^{1}$ (1909), acting on this suggestion, reduced the pigeon's temperature by subcutaneous injections of pyramidon and then injected pneumococci intraperitoneally, with the result that the bird died in a few hours with a marked pneumococcal peritonitis.

These experiments with pyramidon were repeated. A normal pigeon with a cloacal temperature of $106 \cdot 2^{\circ} \mathrm{F}$. was given a subcutaneous injection of $0.45 \mathrm{~g}$. of pyramidon. Two hours and a half later, when its temperature was $101.2^{\circ}$, it was inoculated with four blood agar slope cultures of pneumococcus suspended in saline. The pneumococcus, which was of type II., was originally isolated from a case of pneumococcal meningitis, but had been grown for som months on laboratory media. No attempt was made to heighten its virulence by animal passage. The pigeon died 31 hours later and on post-mortem examination showed an extensive exudate into the peritoneal cavity. The exudate which was swarming with pneumococci, contained a large number of polymorphonuclear leucocytes in various stages of degeneration. There did not appear to be any phagocytosis of pneumococci. The pneumococcus was also recovered in pure culture from the heart blood. The administration of pure culture from the heart blood. The administration of $0.45 \mathrm{~g}$. of pyramidon subcutaneously or the intraperitoneal
injection of the same dose of pneumococci was without any effect on pigeons.

It is well known that one of the effects of a diet of polished rice on birds is to bring about a reduction in the body temperature, though whether by a direct action on the central nervous system or by its retarding effect on all metabolic processes is at present unknown. The reduction in body temperature begins during the first week of the polished rice diet and is progressive, until, when the temperature is $100-101^{\circ}$, the bird dies with the well-known nervous symptoms.

Four pigeons were placed on a diet of polished rice until their temperatures were reduced to between 103-104 after from 21 to 26 days on the rice diet. They were then each inoculated intraperitoneally with six blood agar slope cultures of the pneumococcus, while four control pigeons were similarly inoculated. Death took place in the beri-beric birds in from 9 to 36 hours after the inoculation, while the control birds showed no untoward symptoms. None of the beri-beric birds showed any symptoms of paralysis either before or after the injection of pneumococci. In each instance the beri-beric birds showed a considerable amount of fibrinous exudate in the abdominal cavity, while the pneumococcos was isolated in all four cases in pure culture both from the 